\title{
Digital image-processing techniques for the display of images and modeling of visual perception
}

\author{
TERRY CAELLI \\ The University of Alberta, Edmonton, Alberta, Canada
}

\begin{abstract}
With the rapid increase in power of image-processing technology, applications in the areas of robot and human vision are inevitable. In this review, I consider how such techniques make it possible to study new dimensions of human vision and I suggest explanations of visual phenomena, which, until now, have not been considered in detail.
\end{abstract}

The question as to whether technological advancement precedes scientific insight remains unanswered and, indeed, may be so equivocal as a nonspecific question that it is not worth answering. In the area of visual perception, however, it is certainly the case that specific questions in visual perception, and their answers, have been encountered within the evolution of technological developments. The parallel between models for visual information processing and the evolution of computer architecture in the 1960s and the recent use of linear systems theory to model spatiotemporal vision (Caelli, 1984; Marr, 1982; Watson \& Ahumada, 1985) are but two areas in which developments in technology and science have been used to study vision. The use of point-plot displays to study the visual processing of simple spatiotemporal signals, vector generators, and raster displays for more complex images are examples of new devices that have changed the questions of interest to visual scientists.

There is, however, a deeper parallel between computer vision and human vision: both areas are partitioned into similar topics and hierarchies. The historical evolution of such subareas is more probably due to the earlier psychophysical predelections and methodologies. Table 1 shows a typical representation of areas and associated problems across both disciplines. Such areas are not independent; for example, feature-extraction processes are useful in pattern recognition.

It should also be noted that this division of areas is by no means the only valid one, particularly when specific dependencies exist between areas. For example, the idea that figure/ground perception, or texture segmentation, must precede pattern recognition is not self-evident. Indeed, processes of pattern recognition are intrinsically in-

This research was supported by Grant A2568 from the Natural Sciences and Engineering Council of Canada. Requests for reprints should be sent to: Terry Caelli, Department of Psychology, or Alberta Centre for Machine Intelligence and Robotics, The University of Alberta, Edmon ton, AB, Canada T6G 2E9. volved in aspects of figure/ground segmentation. In this paper, less equivocal definitions of the types of processes and paradigms involved in vision are sought in order to dispel such confusion.

In Table 1, the "top-down" division of visual processes is used to mean that prior knowledge about the environment, signal, or task is required before the perceptual problem can be solved. This is to be contrasted with the "bottom-up" division, for which the processing algorithms, per se, suffice. For example, the detection of a signal (having undergone some transformation) embedded in an image, requires the prior specification of the signal and its possible states. Texture segmentation, by definition, does not require the prior specification of signals (or micropatterns): the segmentation process must produce textured regions of its own accord.

The aim of Sections 1 through 5 is to illustrate some of the paradigms, representations, and models within each of the subareas defined in Table 1. Again, of particular interest is the parallel between developments in vision research and computational vision. Common to all the issues to be discussed is the basic representation of the images to be processed by both systems: a finite resolution or bandlimited achromatic (in this paper) image depicted as a $2^{n} \times 2^{n}$ array of locations (pixels) for which each luminance value is limited to a finite number of (perceptually) resolvable intensities (typically limited to 256 intensities or 8 bits). Clearly the pixel value-to-intensity transformation must reflect the specific psychophysical response function under study. However, general logarithmic or linear transformations are more typically employed when using such digital display devices for human studies. For images subtending a visual angle of $2^{\circ}$ where the (presumed) pixel resolution is approximately 0.5 in., a resolution of $256 \times 256$ pixels would be required. This indicates that the underlying dimensionality of such a stimulus is $256 \times 256=65,536$, a formidable order. Consequently, the aim of most techniques is to reduce the effective dimensionality required to solve specific problems. 
Table 1

Some Fundamental Problems Associated with the Major Areas of Interest in Computational Vision and Visual Perception

\begin{tabular}{|c|c|c|c|}
\hline Section* & $\begin{array}{l}\text { Fundamental } \\
\text { Problems }\end{array}$ & $\begin{array}{l}\text { Computational } \\
\text { Vision }\end{array}$ & $\begin{array}{l}\text { Visual } \\
\text { Perception }\end{array}$ \\
\hline \multicolumn{4}{|l|}{$\begin{array}{l}\text { Bottom-Up } \\
\text { Processing }\end{array}$} \\
\hline 1 & $\begin{array}{l}\text { What is the best } \\
\text { representation for an image } \\
\text { to satisfy specific biologi- } \\
\text { cal or computational } \\
\text { constants? }\end{array}$ & $\begin{array}{l}\text {-Image domain: } \\
\text { predictive compression } \\
\text {-Orthogonal decomposition } \\
\text {-Fourier representations }\end{array}$ & $\begin{array}{l}\text {-Retinal and cortical } \\
\text { encoding principles } \\
\text {-Psychophysical } \\
\text { perceptive fields }\end{array}$ \\
\hline 2 & $\begin{array}{l}\text { What processes lie behind } \\
\text { texture segmentation or } \\
\text { figure/ground perception? }\end{array}$ & $\begin{array}{l}\text {-Image segmentation: } \\
\text { region grouping, } \\
\text { surface gradient } \\
\text { extraction }\end{array}$ & $\begin{array}{l}\text {-Figure/ground perception } \\
\text {-Texture discrimination }\end{array}$ \\
\hline 3 & $\begin{array}{l}\text { What image or pattern } \\
\text { features are ideal for the } \\
\text { encoding of spatial } \\
\text { structures? }\end{array}$ & $\begin{array}{l}\text {-Edge extraction } \\
\text {-Pattern feature codes } \\
\text { for object recognition }\end{array}$ & $\begin{array}{l}\text {-Feature detectors } \\
\text { for pattern recognition } \\
\text {-Edge encoding } \\
\text {-Criteria for } \\
\text { perceptual form }\end{array}$ \\
\hline 4 & $\begin{array}{l}\text { What processes best repre- } \\
\text { sent the detection of signals } \\
\text { embedded in images? }\end{array}$ & $\begin{array}{l}\text {-Pattern recognition via } \\
\text { transform methods, } \\
\text { feature correlation, } \\
\text { matched filtering }\end{array}$ & $\begin{array}{l}\text {-Pattern recognition: } \\
\text { correlated outputs of } \\
\text { feature analyzers, } \\
\text { Fourier representations }\end{array}$ \\
\hline $\begin{array}{l}\text { Top-Down } \\
\text { Processing }\end{array}$ & $\begin{array}{l}\text { How do visual systems } \\
\text { recognize patterns under } \\
\text { translations, rotation, and } \\
\text { size changes? }\end{array}$ & $\begin{array}{l}\text {-Invariance coding } \\
\text {-Circular harmonic } \\
\text { decomposition } \\
\text {-Log-polar methods }\end{array}$ & $\begin{array}{l}\text {-Problem of stimulus } \\
\text { equivalence } \\
\text {-Recognition under } \\
\text { transformations }\end{array}$ \\
\hline
\end{tabular}

*The section number refers to the section of this paper in which the topic is discussed.

\section{FUNDAMENTAL PROBLEMS}

\section{Issues of Image Encoding}

One of the most fundamental problems in human vision is that of determining how the observer encodes an image. We can restrict our definition of an image to, for instance, a $256 \times 2568$-bit format within the foveal region; this constitutes up to 524,288 bits of information, which is a large amount to be processed within a saccade. Solutions to this problem generally fall into one of two schools: explanations based on image models (in the image domain) or those based on representations in some transform domain. In both cases, however, the aim is to uncover encoding units of much lower dimensionality than defined by the pixels alone.

The predominant image-domain model, in biological vision, is that based on the perceptron, a model formalized by Minsky and Papert (1969). In this model, spatial information is envisaged to be encoded by large numbers of "receptive fields," or, in the psychophysical world, those "perceptive fields" that cover more than one pixel. The responses of such detectors are determined by the cross-correlation (or convolution, depending on how the detector profile is defined) of the detector's profile with the incoming image. Being localized in space, such a detector's response is defined by:

$$
R_{i}\left(x_{0}, y_{0}\right)=\int_{-\infty}^{\infty} d_{i}(\alpha, \beta) I\left(\alpha-x_{o}, \beta-y_{0}\right) d \alpha d \beta,
$$

where $d_{i}(\alpha, \beta)$ corresponds to the detector centered at $\left(x_{0}, y_{0}\right), I(x, y)$ corresponds to the image, and $(x, y)$ to a locally Euclidean retinotopic coordinate system. If we consider many such detectors centered at $\left(x_{0}, y_{0}\right)$, then the linear perceptron model allows an encoding of the intensity at $\left(x_{0}, y_{0}\right)$ by

$$
I\left(x_{0}, y_{0}\right)=\sum_{i=1}^{n} R_{i}\left(x_{0}, y_{0}\right) .
$$

Whether it is at the retinal or cortical levels of analysis, this approach proposes that, relative to sampling consideration (see Geisler \& Hamilton, 1986), such detectors are distributed over the image such that the response at any position $(x, y)$ is determined by:

$$
R_{i}(x, y)=\int_{-\infty}^{\infty} \int_{i}(\alpha, \beta) I(\alpha-x, \beta-y) d \alpha d \beta
$$

In this case, any pixel intensity is usually envisaged to be "internally" encoded by

$$
I(x, y)=\sum_{i=1}^{n} R_{i}^{\prime}(x, y),
$$




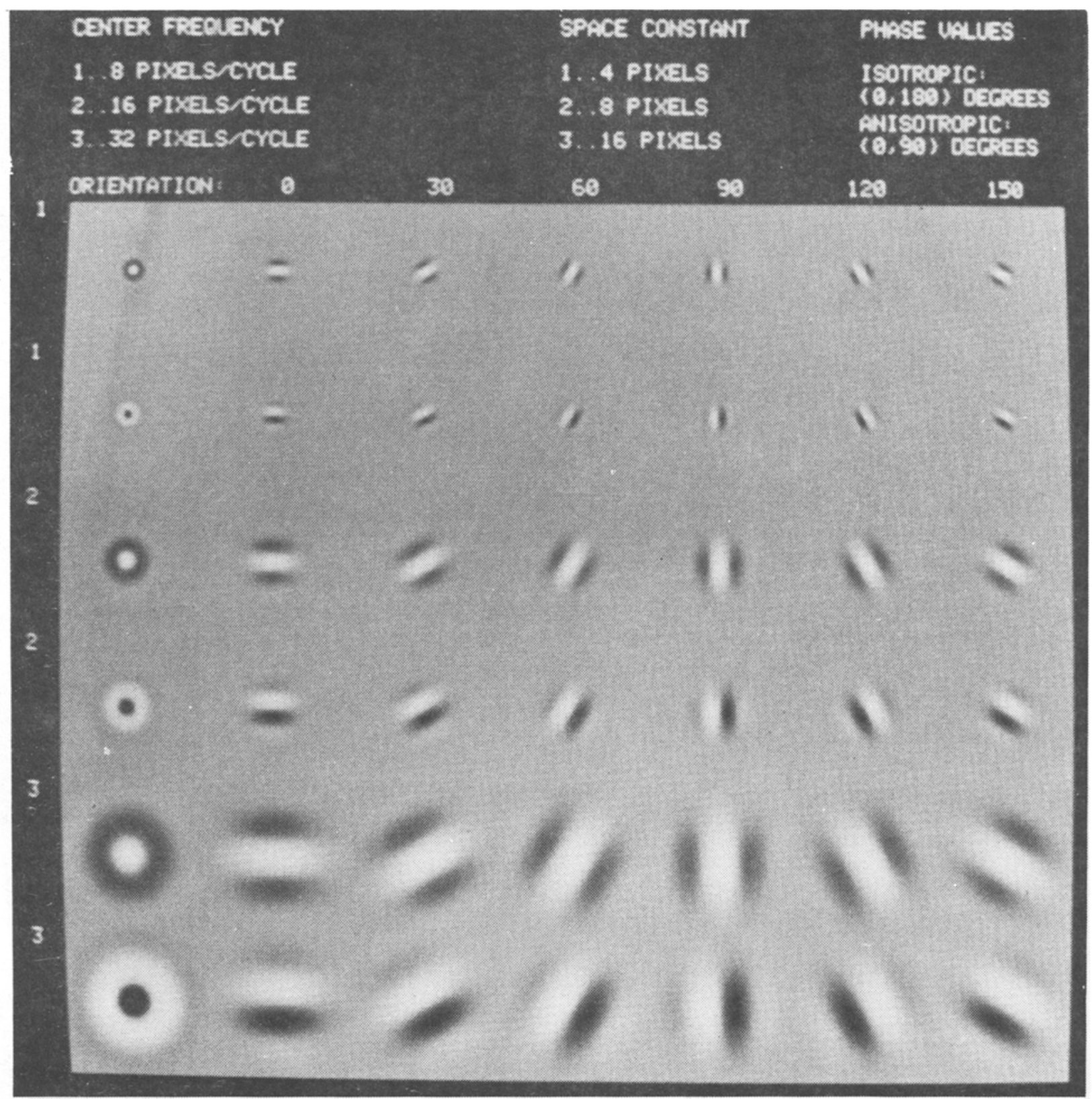

Figure 1. Sets of hypothetical detectors corresponding to feasible retinal ganglion cell receptive fields (Cohumn 1) and simple cells (Cohumns 2-7). Apertures (space constants), center frequencies, and phase values are relative to an underlying $64 \times 64$ pixel format. The correlation (zero shift) between even and odd detectors was zero and between neighboring orientations was no better than 0.5 . The orientation-specific detectors are spaced at $30^{\circ}$ apart and have a fixed-orientation turning width of $\pm 15^{\circ}$ over all three center radial frequencies. The bandwidths increase with increases in center frequencies.

where $R^{\prime}$ is seen as a nonlinear transform of the linear response function $R$ (see, e.g., Wilson \& Gelb, 1984, for an example). The problem with this representation is that the number of detectors required to "construct" the image is as large as the number of pixels, as we will see in the case of the transform models. For example, if we employed detectors consistent with retinal ganglion receptive fields (Figure 1, left column) of different sizes, then the number of such detectors required to reconstruct the image faithfully would exceed the number of pixels (see also Figure 2). Even recent attempts to represent such detectors as localized in both image and frequency domains with two-dimensional Gabor profiles (Daugman, 1984; Papoulis, 1968) do not solve this problem of high dimensionality, as will be seen.

The second approach to image representation involves the proposal that images are encoded in terms of their twodimensional Fourier components and that such compo- 


\section{FACE}
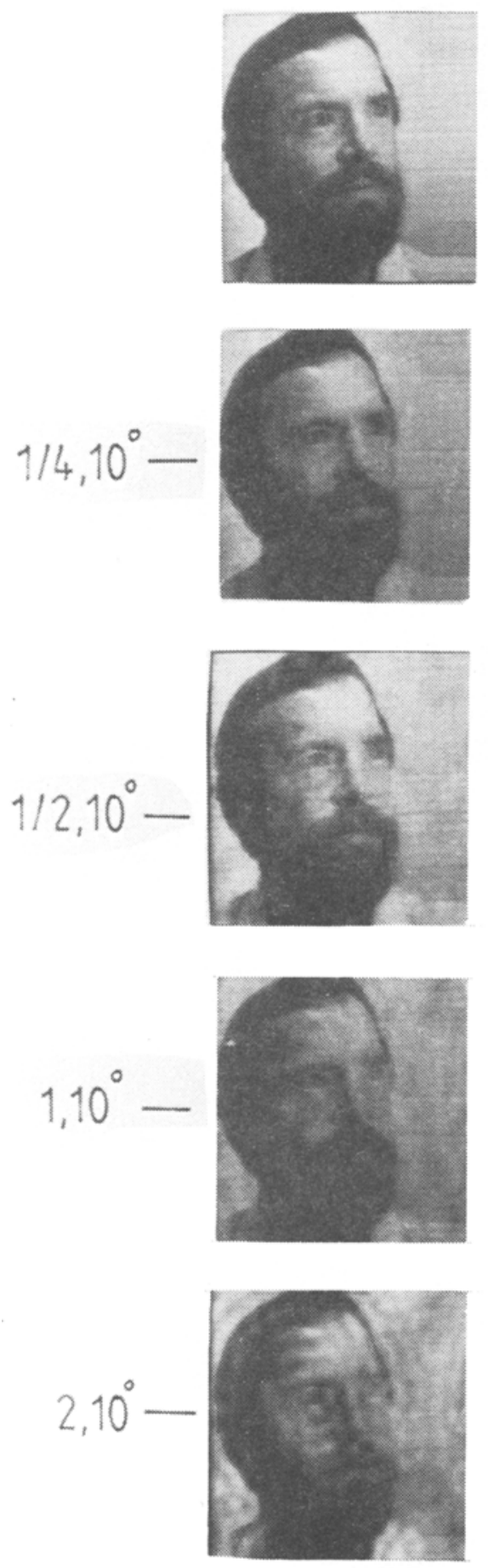
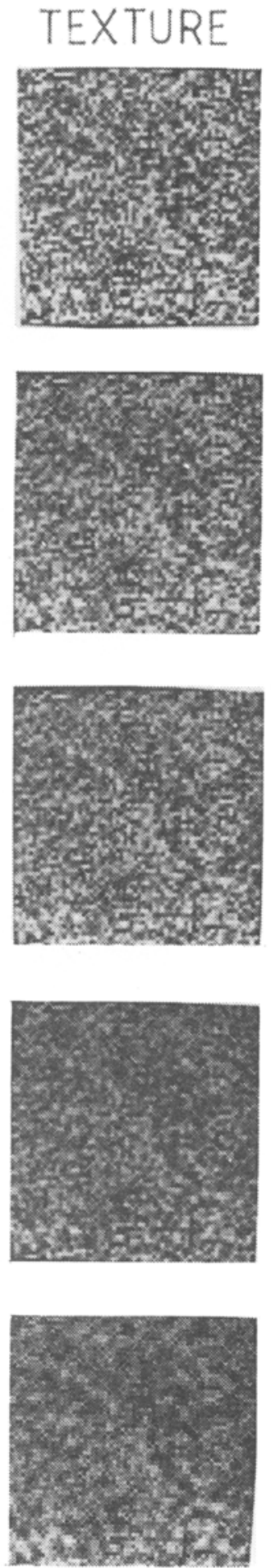
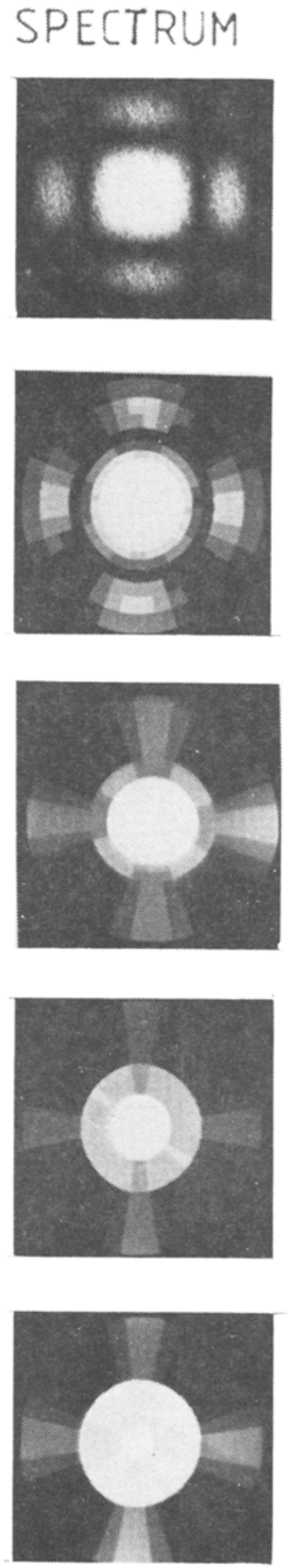

Figure 2. Top row shows two original images digitized as $256 \times 2564$ bit pixels and the amplitude spectrum for the texture. Rows 2 through 5 represent the inverse Fourier transforms of the amplitude-averaging process illustrated for the texture case in the right-hand column. The unit radial frequency widths (octaves: RF) and orientation tuning (in degrees) are shown on the left (RF, $\theta$. From "Coding images in the frequency domain: Filter design and energy processing characteristics of the human visual system" by T. Caelli and M. Hübner, 1983, IEEE Transactions on Systems, Man, and Cybermetics, 13, 1018-1021. Reprinted with permission. 
nents form discrete numbers of "channels" (see R. de Valois \& K. de Valois, 1980; Sekuler, 1974). Usually the outputs of such channels are restricted to (scalar) energy values (Braddick, Campbell, \& Atkinson, 1978) and, as such, by no means uniquely encode the incoming image. In fact, using this criteria, Caelli and Hübner (1983) found that at least 90 channels would be required in the two-dimensional frequency domain (without even considering phase encoding) to capture the input signal (Figure 2). Although this may be possible for a parallel-processing biological system, it does not make good science to have an encoding model with at least 90 parameters.

Such results suggest that perceptrons do not produce a more efficient image code than do the individual pixels and that if efficiency is required, then one may as well remain in the image domain. In contrast to this situation, there are many techniques in digital image processing that produce considerably more efficient image-encoding strategies. For illustrative purposes, we consider one: predictive compression.

Predictive compression differs from the above approaches insofar as it does not posit the existence of fixed, invariant detectors that encode the image. Rather the encoder is a correlator, which determines the degree to which an individual pixel can be predicted from its neighbors (see Rosenfeld \& Kak, 1982, for more details). Since nonwhite (nonrandom) images, by definition, carry highly correlated information, this technique can produce considerable reduction in bit codes (Caelli \& Hübner, 1984).

The technology required to study issues in visual or computer image encoding includes standard imageprocessing systems that have digitizing cameras $(512 \times 512$ 8-bit frame "grabber"), frame memories, and highquality (raster) monitors. In addition, a sufficiently fast processor (or dedicated peripheral device) is required to compute the convolutions for the perceptron-based models. The detector response functions (Equation 3) can also be enacted by fast Fourier transform techniques since Equation 3 is equivalent to:

$$
R_{i}(x, y)=F^{-1}\left\{F\left(d_{i}(x, y)\right) \cdot F(I(x, y))\right\},
$$

where $F$ and $F^{-1}$ correspond to Fourier and inverse Fourier transforms, respectively. Discrete forms of the Fourier transform are enacted by the well-known CooleyTukey method (Cooley \& Tukey, 1965), which, for large kernels (detector profiles), is considerably faster than enacting Equation 3 directly. However, it should be noted that with the increasing speed, versatility, and "parallelness" of array processors, this may not be the case in the near future.

As is evident in Figure 2, one direct psychophysical paradigm for studying the superthreshold sensitivity to the compression or detection of spatial information is that of discrimination. If a specific encoding dimension is critical to the perception of an image, then the perceived difference between an original image and a version com- pressed (by a fixed amount) along this dimension should be greater than parametrically equivalent compressions along others. A good example of this is compression in Fourier power compared with phase, where it has been conclusively shown (Caelli, 1983; Caelli \& Bevan, 1983) that phase is the more important processing parameter. In turn, it can also be shown that phase compressions have more deforming effects on the image luminance profiles; thus, Caelli and past collaborators have concluded that it is the pixel, or image-domain, parameters, that are of paramount importance, even after 20 years of attempts to show that visual encoding may be accomplished in a transform domain. This does not imply that the visual system is not differentially sensitive to different luminance gradients. As shown in Figure 3, it is clear that bandpass components contain the more perceptually salient features of an image, since the predominant face is the one belonging to that range when two images are digitally superimposed (see Caelli \& Yuzyk, 1985 , for more details). Indeed, such demonstrations show that image encoding cannot be divorced from the selective attention of the visual system for specific features, such as edges (this topic is covered in Section 3).

\section{Image Segmentation:}

Figure/Ground Processing?

Although image encoding is concerned with image representation in general, image or texture segmentation research has the specific aim of developing models for how biological and computer visual systems may segment arbitrary images into regions that are defined by their "granularity" or surface properties. Texture segmentation also differs from pattern recognition insofar as no a priori information about the segmentation areas is supplied before segmentation occurs.

Psychophysical studies of texture segmentation have usually been restricted to artificial textures composed of pseudowhite noise or textures generated from micropatterns (Beck, 1966; Julesz, 1962, 1981). In all such studies, the aim has been to enumerate the specific types of texture properties that determine perceptual segmentation, and, over the past 20 years, it has become apparent that the process seems to occur in the visual system through the registration of differences between the outputs of detectors, sometimes referred to as "textons" (Julesz, 1981). The idea that classification of textured regions comes about by such a process was studied earlier by Richards and Pollit (1974) with one-dimensional granularities and recently by Caelli (1985). One typical computational scheme for texture segmentation and an example are shown in Figure 4.

From the example in Figure 4, three processes seem necessary to represent how segmentation may be accomplished: (1) a form of convolution, where specified detectors are cross-correlated with the incoming texture, as in Equation 3; (2) a form of impletion, or perceptual "relaxation," where strong and contiguous responses are allowed to spread while isolated or weak detector 


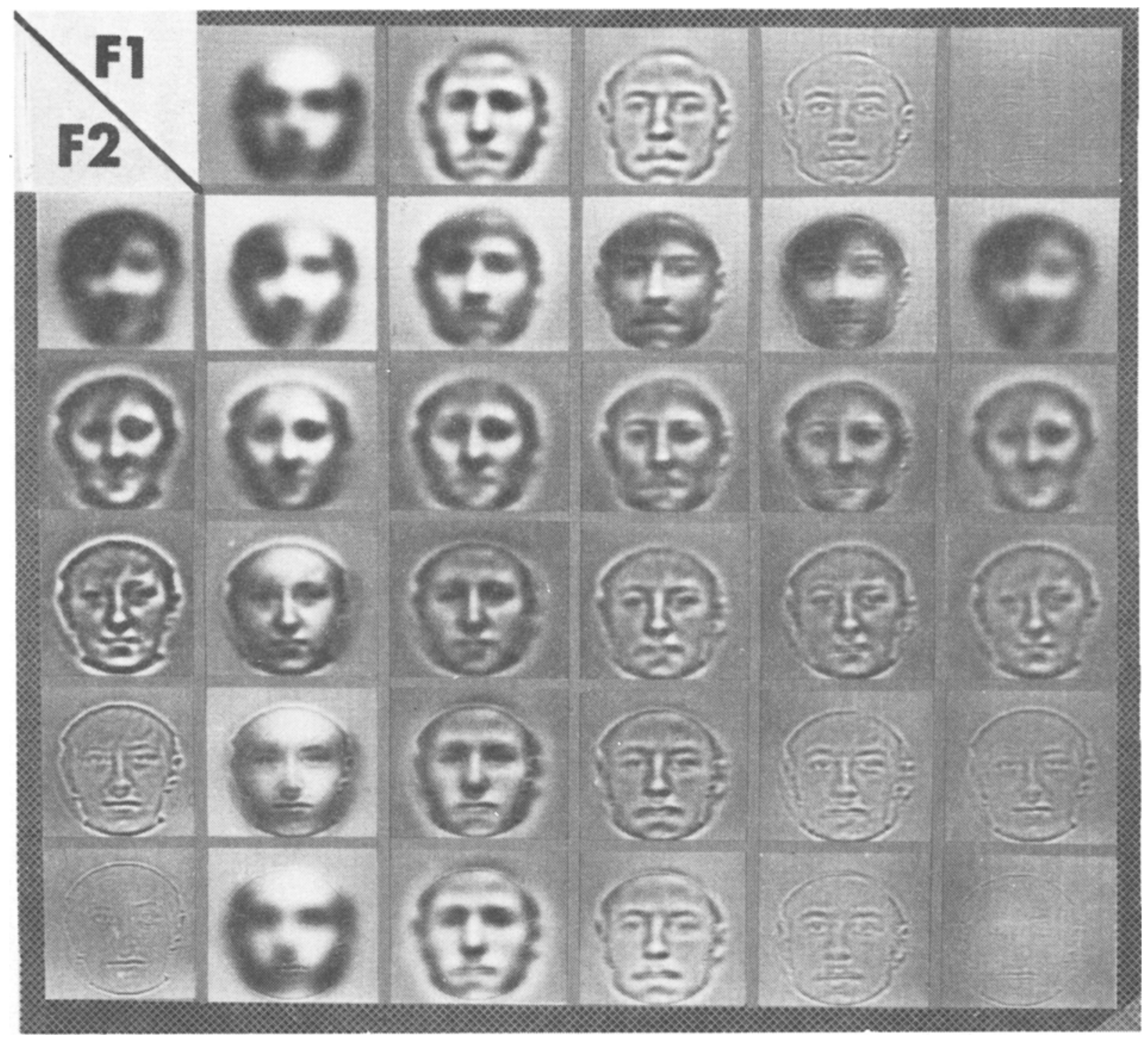

Figure 3. Face-face montage. Row 1 consists of Face 1 images after filtering $(0,8,16,32,64$ picture cycles). Column 1 consists of Face 2 images after filtering $(0,8,16,32,64$ picture cycles). The remaining images are the result of factorially combining filtered Face 1 and Face 2 images.

responses are inhibited; (3) grouping, where the vector responses of each position (pixel) are correlated such that positions with similar detector outputs are grouped into the same textured region. Below a certain correlation threshold, the positions are classified into different regions.

Questions about the number and shape of the detectors involved are important; however, their answers do not solve the problem of texture segmentation. Indeed, Figure 4 illustrates one difference between computer vision and much of behavioral research insofar as the former requires that a full segmentation algorithm be supplied. Even the issue of detector specification can be clarified if one adopts the objective definition of orthogonality; that is, two detector profiles $\left(d_{i}, d_{j}\right)$ are orthogonal if, and only if, their scalar product is zero:

$$
d_{i} \cdot d_{j}=\sum_{x} \sum_{y} d_{i}(x, y) \cdot d_{j}(x, y)=0 .
$$

Earlier attempts to define the basic set of orthogonal detectors (see, e.g., Caelli, Julesz, \& Gilbert, 1978) did not use this form of independence, at least as an objective benchmark. Indeed, claims that such texture features as crossing-lines and terminators are distinctively different from orientated edge or bar detectors (Julesz, 1981; Treisman \& Gelade, 1980) are not consistent with the above definition of orthogonality, and are not even necessary to predict the very results used to argue for such features via Equation 3 (see Caelli, 1985, for details). Such distinctions simply demonstrate the differences between descriptions developed for spatial codes in the vernacu- 

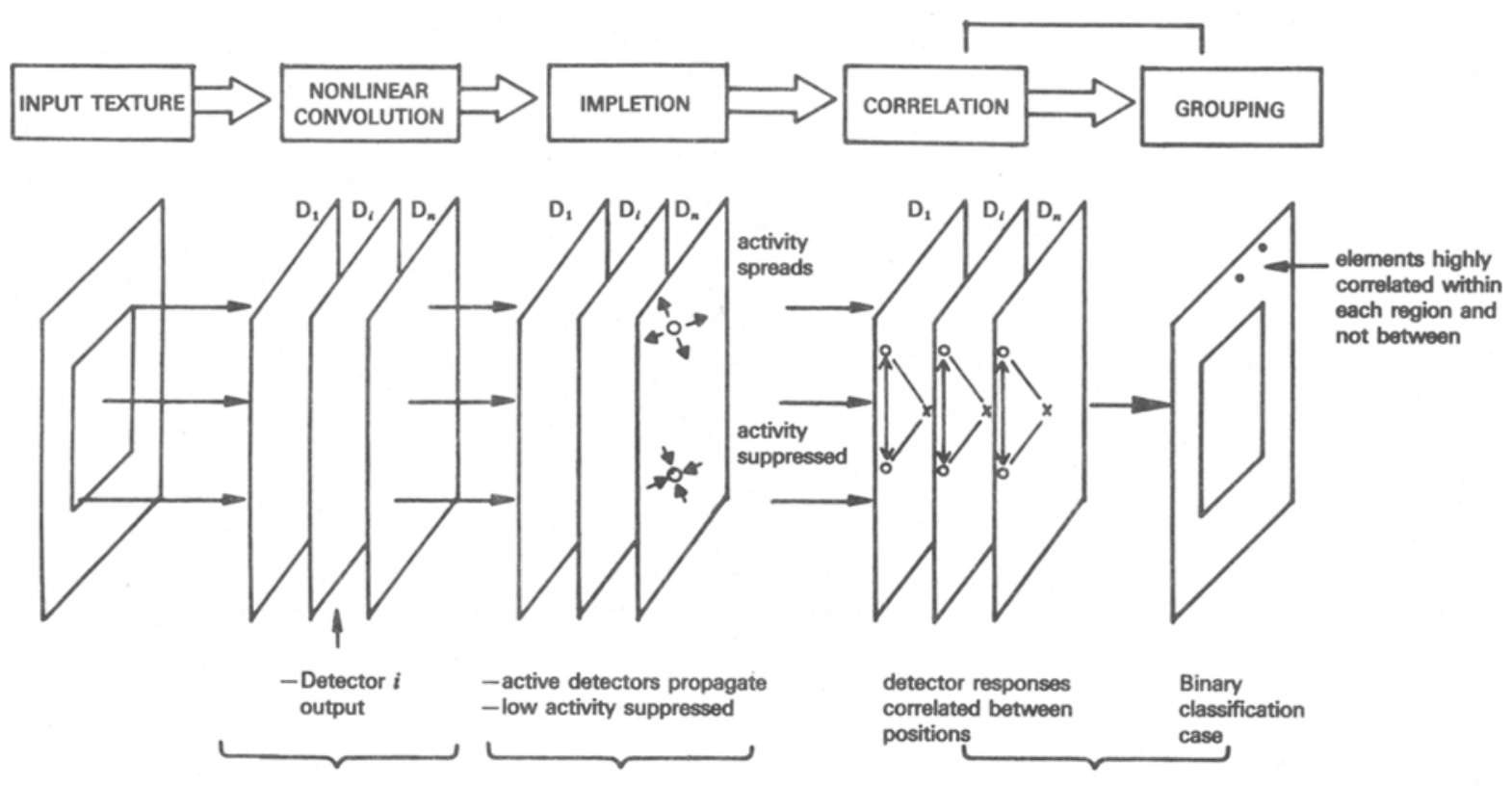

Heuristic 2:

Heuristic 3:

Heuristic 1:

detection of textons as nonlinear convolution kernels: lines, edges, Gabor signals, crossings, terminators, etc. selective activity of textons preattentive decision process

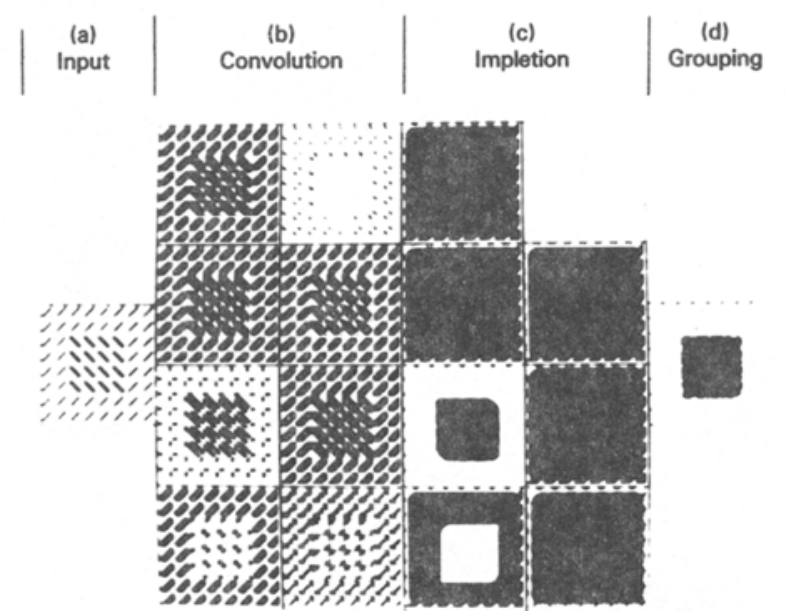

Figure 4. Texture segmentation model proposed in "Three processing characteristics of visual texture segmentation" by T. Caelli, 1985, Spatial Vision, 1, 19-30 (reprinted with permission). (a) Basic detectors, their spatial coupling, and selectivity, which result in the "filling in" of textured regions and segmentation effects. (b) An exomple in which the input texture (left) is processed by eight edge/bar detectors (outputs represented in the first two columns), after which impletion is activated to result in "filling in" by active units and suppression of inactive units. The final output represents the grouping of positions according to the detector outputs (from Caelli, 1985). 
lar and objective formal languages as suggested by Equation 5.

The technological requirements for texture displays and experiments are dependent upon the type of textures under investigation. Point or vector-type black/white micropattern textures can be generated on point-plot CRTs, as in earlier displays (see, e.g., Caelli et al., 1978). However, fully gray-scaled, or even chromatic, textures require raster monitors and image-processing systems as described in the previous section.

\section{Image-Feature Extraction}

Consistent with the view that the critical information in images for pattern recognition, classification, and so forth lies in what can be loosely termed the image's "structural features," attempts have been made over the past century to define such image components in an objective way. Similarly, much research has been done to determine whether the visual system is particularly sensitive to a subset of such features and whether such features are sufficient to predict many aspects of spatial vision.

From the detection of Gaussian windowed gratings (Watson, Barlow, \& Robson, 1983) to the masking of one image by another (Caelli \& Yuzyk, 1985), it is clear that the human visual system is more sensitive to image bandpass information than is physically present and measured by the image power spectrum. That is, image energy usually decreases with increases in spatial frequency (luminance gradients); however, visual sensitivity is typically "inverted-U" shaped with pure grating patterns (as in contrast sensitivity measures) or images in general. This follows naturally from the predominance of known detectors within the vertebrate visual system that have little response to constant luminance and specific sensitivities to edges, bars, and so forth (see Hubel \& Wiesel, 1977, for a review).

Techniques for edge extraction are consequently based upon at least some form of bandpass filtering and thresholding operation. The choice of bandpass is usually made to minimize the degree of high-frequency edge "noise," but must be sufficiently highpass to extract adequate luminance gradients (detail). One popular way of enacting such a bandpass filter is to use a $\nabla^{2} G_{\alpha}$ operator consisting of a Gaussian lowpass filter whose bandwidth is determined by $\alpha$ (see Caelli \& Nagendran, in press). By definition, the blurring process $\left(G_{\alpha}\right)$ attenuates the high-frequency components, and the $\nabla^{2}$ operator, being a Laplacian differential operator, enhances the remaining detail. This operation is analytically defined by

$$
\nabla^{2} I=\frac{\partial^{2} I}{\partial x^{2}}+\frac{\partial^{2} I}{\partial y^{2}},
$$

which, in finite-difference form, is equivalent to crosscorrelating the image with the kernel:

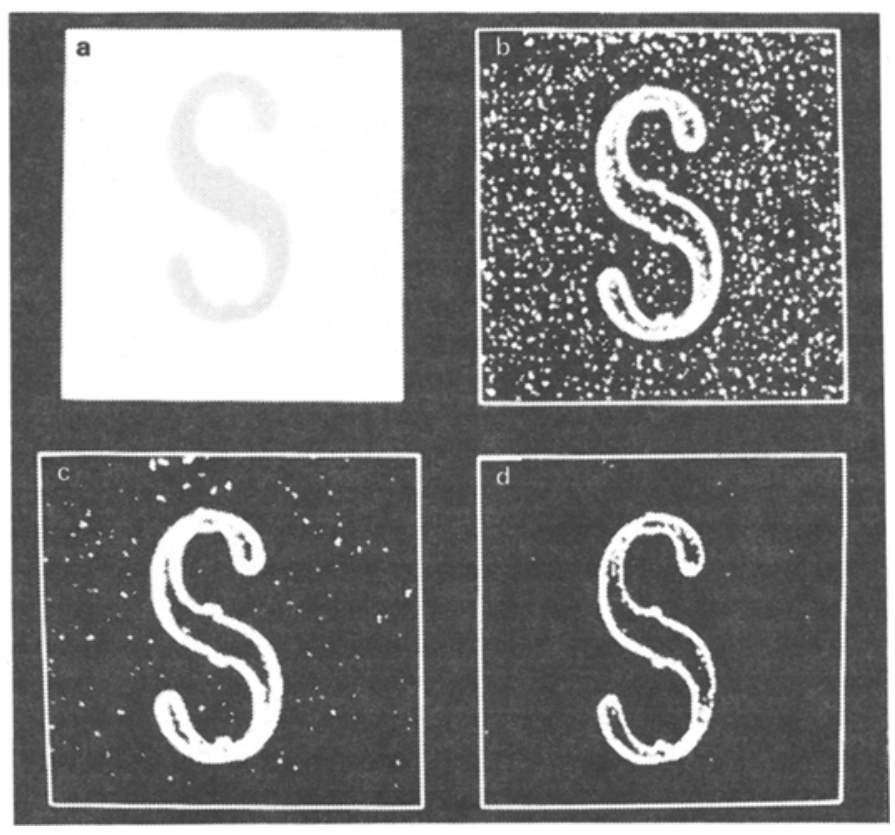

Figure 5. Input signal (a) and the outputs of two different edge extractors (b and c) determined by two difierent $\nabla^{2} G_{\alpha}$ operators (band-poss filters). Here, $\alpha$ corresponded to 4 - and 6-pixel space constants, respectively. Figure 5d corresponds to the ANDed version of these two edge images. 


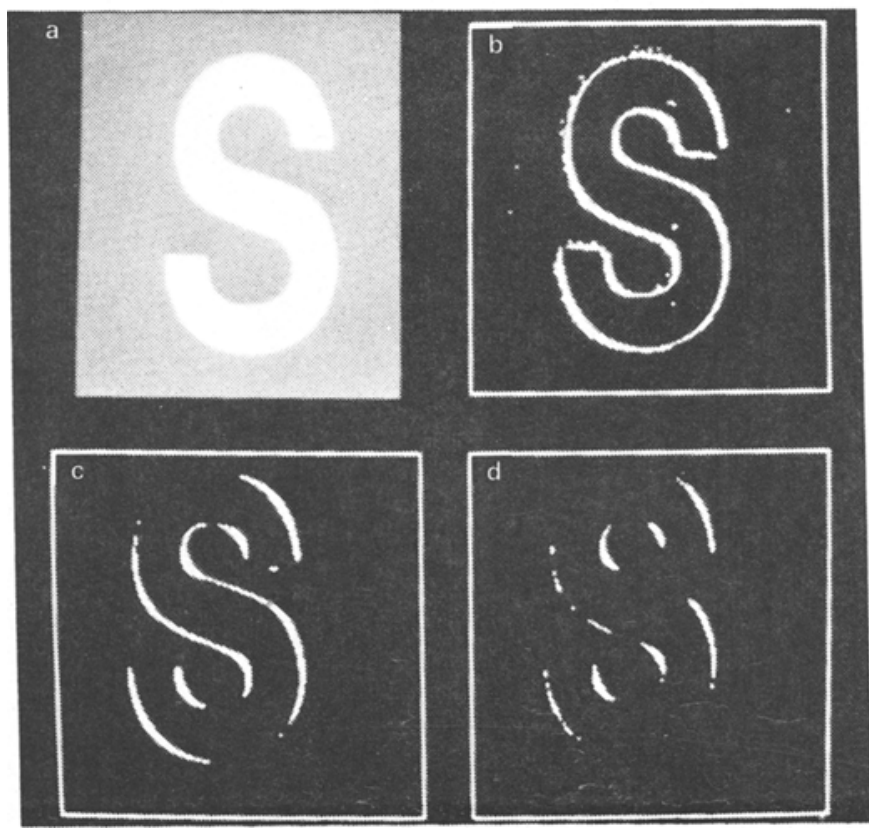

Figure 6. Figure 6 is the same as Figure 5, except for the use of relaxation, where only regions of high edge density are retained in different degrees. Here, b, c, and d cerrespond to edge-only images with no extra constraints (top right), only retaining edge points after averaging and thresholding within 4 and 12 pixel radit.

$$
\hat{\nabla}^{2} \equiv\left[\begin{array}{rrr}
0 & -1 & 0 \\
-1 & 4 & -1 \\
0 & -1 & 0
\end{array}\right]
$$

Indeed, when two such $\hat{\nabla}^{2}$ s are put together (one at $45^{\circ}$ to the other), one sees the usual lateral inhibition (L.I.) operator:

$$
\text { L.I. } \equiv\left[\begin{array}{rrr}
-1 & -1 & -1 \\
-1 & 8 & -1 \\
-1 & -1 & -1
\end{array}\right]
$$

which, again, serves to differentiate the image. An example of edge extraction, based on $\nabla^{2} G_{\alpha}$, is shown in Figure 5 in a more elaborate form. Figures $5 \mathrm{~b}$ and $5 \mathrm{c}$ represent the thresholded outputs of two $\nabla^{2} \mathrm{G}_{\alpha}$ filters at two different values of $\alpha$ : the Gaussian space constant is set at 4- and 6-pixel widths. All edge responses greater than $\nabla^{2} G_{\alpha}=0$ are retained. Figure $5 d$ represents the logical $A N D$ between these two edge outputs, and the effect is to retain the stronger edges and, thus, suppress "edge noise" (Caelli \& Nagendran, in press).

By introducing an operation called "relaxation," such edge-only information can be reduced to edge regions of high density (as shown in Figure 6). Such relaxation techniques have wide use in computer vision and are based upon an iterative scheme that enhances edge responses if neighborhood responses are strong and that inhibits the response if neighborhood outputs are weak (see Hummel \& Zucker, 1983).

There are many other versions of edge operators in use, although most involve some form of image differentia- tion (see Rosenfeld \& Kak, 1982) and thresholding operation. The two most common edge-decision criteria are the simple thresholding operation illustrated in Figures 5 and 6 and the use of zero-crossings in the $\nabla^{2} G$ images. Indeed, the psychophysical evidence for the perceptual uniqueness of any of these criteria is lacking (Caelli \& Yuzyk, 1986; Watt \& Morgan, 1985).

These operations are simple examples of imageprocessing techniques that may reflect human visual information-processing characteristics and offer new insights into feature encoding. For example, the pattern features for the character " $S$ " shown in Figure 6 are not derived from an explicit list of curvature detectors, but rather from a process that results in these critical features for a specific pattern. As will be shown in Sections 4 and 5 , this process approach to feature generation significantly decreases the dimensionality of the pattern-recognition problem.

Image-processing systems not only allow one to extract such edge-only images quickly by dedicated pipe-line pixel processors (see Caelli \& Nagendran, in press), but also enable more realistic experiments on feature processing. One example is the alignment of image-edge information with an original image where, at raster speed (every 33 msecs), one can move (pen and scroll) the edge version over the original until subjective alignment is reached (see Caelli \& Yuzyk, 1986, for details).

\section{Pattern Recognition: Detecting \\ Signals in Images}

The division in Table 1 between "bottom-up-" and "top-down" processing is to be interpreted in terms of 
the degree to which prior knowledge is introduced into the solution algorithms. In the three topics briefly discussed already, the only "prior knowledge" involved was the computational processes or image-representation strategies; however, with pattern recognition (Section 4) and invariance coding (Section 5), increasing amounts of prior knowledge are required. This knowledge controls the processing and, thus, is termed "top-down."

The simplest example of pattern recognition involves
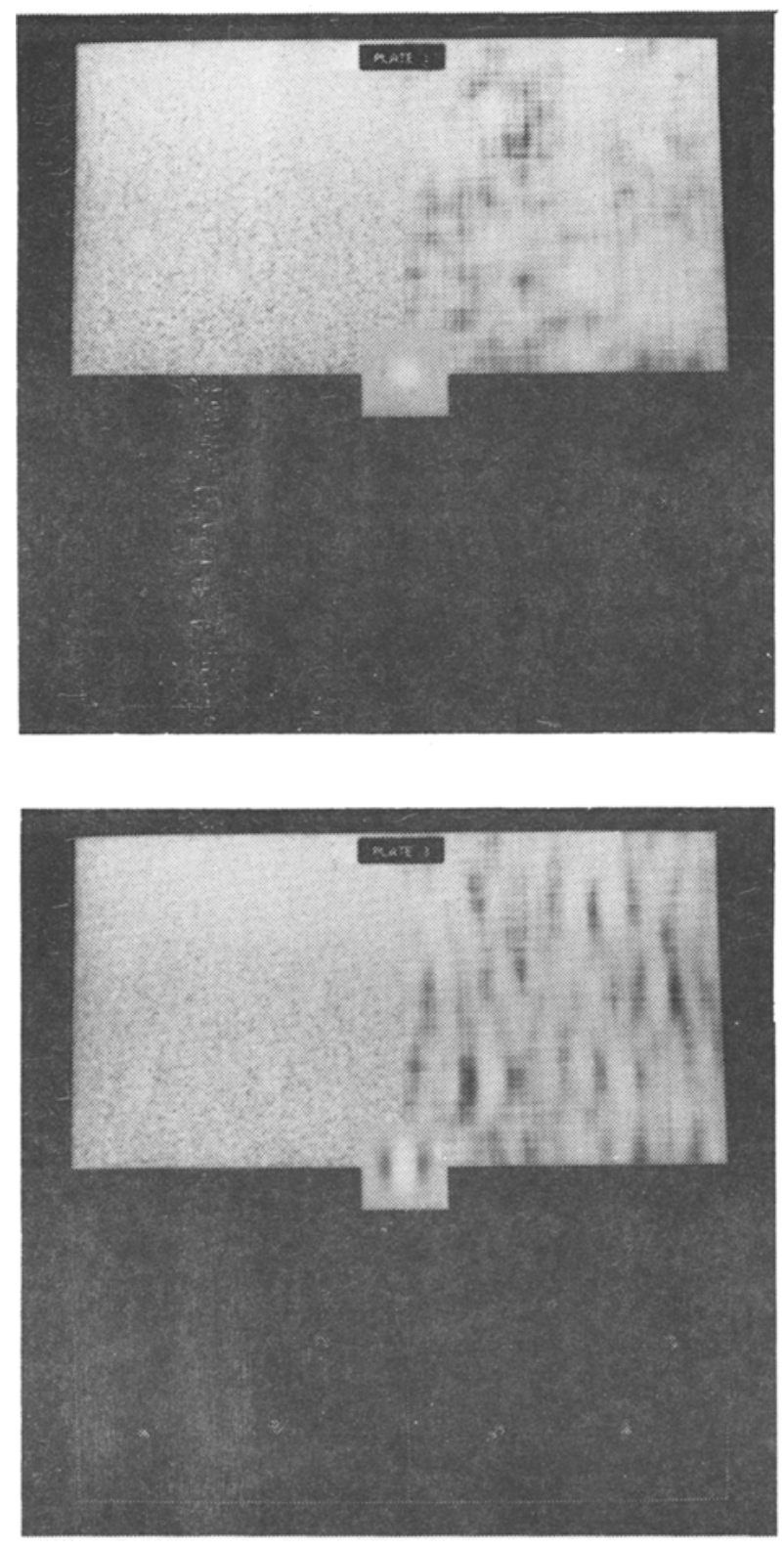
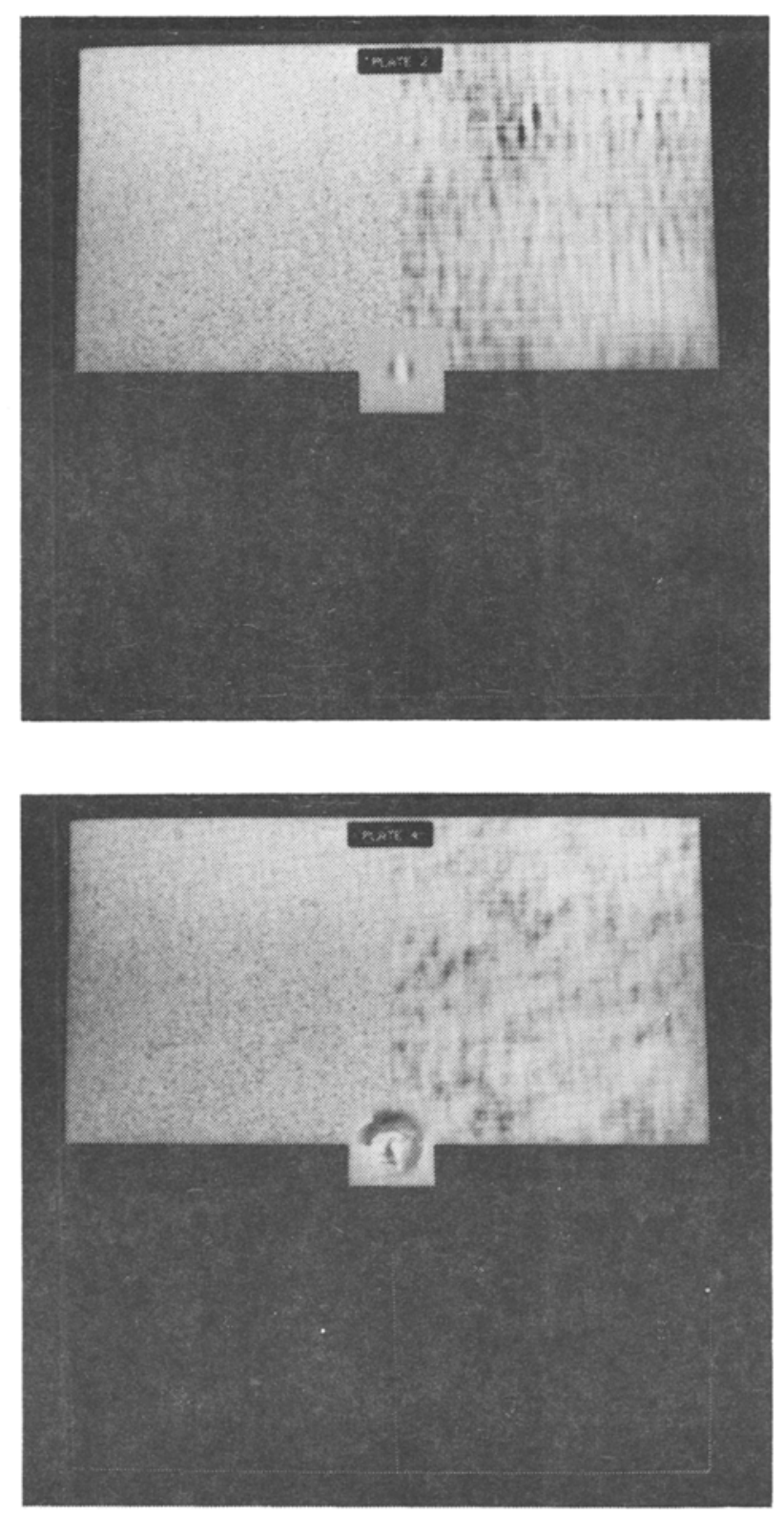

the detection of a signal $f(x, y)$ embedded in an image $g(x, y)$, where

$$
g(x, y)=f(x, y)+n(x, y),
$$

for $n(x, y)$ corresponding to the background. Here, the prior information consists of at least some signal and background specifications, and the question is what filter (of $g$ ) best reveals the presence of $f$ in the image (the "matched filter problem"; see Rosenfeld \& Kak, 1982).

Figure 7. The normalized cross-correlator: Top left images show image with the center (small) signal embedded, and top right images show the normalized cross-correlation output image. Here the brightness indexes the likelihood of the signal's presence. Bottom images show performance of two ohservers in localiving such signals using a "mouse." The densities are derived from 200 trials per observer. From "On the localization of signals in images" by T. Caelli and M. Nawrot, 1986, manuscript submitted for publication. Printed with permission. 
The solution to this problem is determined from what is termed the Cauchy-Schwarz inequality (see Hall, 1979, p. 481); that is, the cross-correlation between $f$ and $g$ is upper-bounded by:

$$
\begin{aligned}
C_{f g}(x, y) & =\int_{-\infty}^{\infty} \int_{\infty} f(\alpha, \beta) \cdot g(x+\alpha, y+\beta) d \alpha d \\
& \leq \sqrt{\int_{-\infty}^{\infty} f^{2}(\alpha, \beta) d \alpha d \beta \cdot \int_{-\infty}^{\infty} g^{2}(x+\alpha, y+\beta) d \alpha d \beta} .
\end{aligned}
$$

If the signal energy $\left(E_{f}\right)$ is defined by

$$
E_{f}=\int_{-\infty}^{\infty} \int^{2}(\alpha, \beta) d \alpha d \beta
$$

and the equivalent image energy is defined by

$$
E_{8}(x, y)=\int_{-\infty}^{\infty} g^{2}(x+\alpha, y+\beta) d \alpha d \beta
$$

then Equation 10 becomes

$$
\frac{C_{f g}(x, y)}{\sqrt{E_{\mathrm{g}}(x, y)}} \leq \sqrt{E_{f}}
$$

with equality if, and only if, $f=\lambda g$ (Rosenfeld \& Kak, 1982).

The normalized cross-correlation images defined by Equation 11 are illustrated in Figure 7. A cross-correlator determines the likelihood of the signal's presence over the full image: both the what and the where. When $n(x, y)$ in Equation 9 is white-noise, having (1) a flat power spectrum, (2) random phase spectrum, and (3) a Gaussian pixel histogram, then $E_{g}$ is constant and the crosscorrelator is optimal for detection (Rosenfeld \& Kak, 1982).

When the background [ $n(x, y)$ in Equation 9] becomes more variable (neighborhood pixel variances and/or means increasingly vary), the optimality of Equation 11 decreases and additional filters have to be developed to improve detection. For example, in a recent paper Liu and Caelli (in press) estimated a bandpass filter $(h)$ that minimizes the difference between the signal autocorrelation $\left(C_{f f}\right)$ and cross-correlation $\left(C_{f_{g}}\right)$ images:

$$
\min _{h} \sum_{x} \sum_{y}\left\{C_{f f}-h^{*} C_{f b}\right\}^{2}
$$

where $h$ is a Weiner filter as it is derived from a least squares constraint (Rosenfeld \& Kak, 1982). In all such cases, it seems apparent that emphasizing edges (as $h$ does, while also attenuating $n$ ) increases signal detectability.

Psychophysical procedures for investigating such issues require image-processing systems that enable one to digitally add small signals (e.g., $64 \times 64$ pixels) to larger images (e.g., $512 \times 512$ ). Various localization response measuring techniques can be used, including forced-choice or search procedures, where the observer moves a mouse
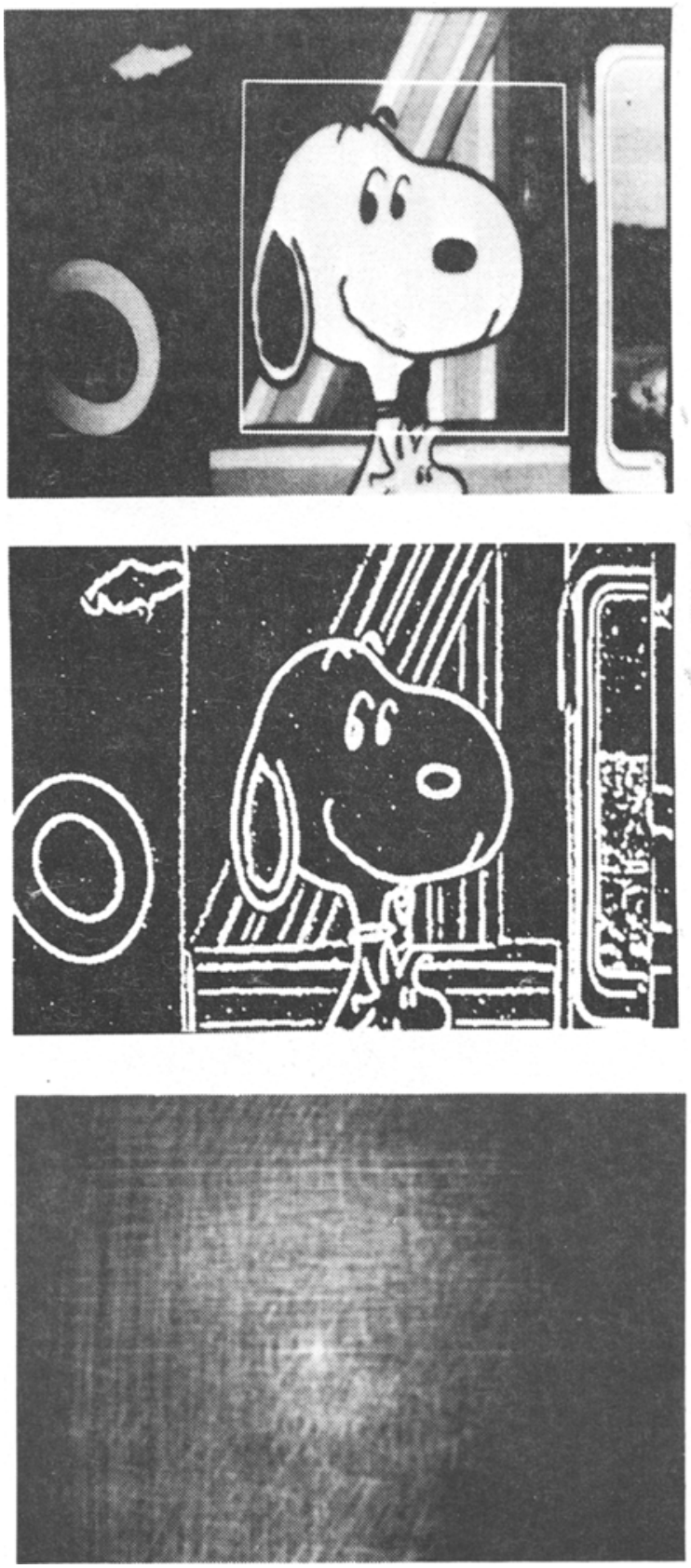

Figure 8. Edge-only cross-correlation where the signal (top) is cross-correlated with the image in edge-only form. Note the sharpness of the cross-correlation output (bottom).

about the image to locate the signal (see Burges \& Ghandeharian, 1984).

Current models for human signal detection center on the concept that pattern recognition occurs by the enactment of some process like Equation 11 with many different versions of $f$ and $g$ as determined from the outputs of specific detector (receptive field) profiles. This multivariate classification would presumably improve signal 

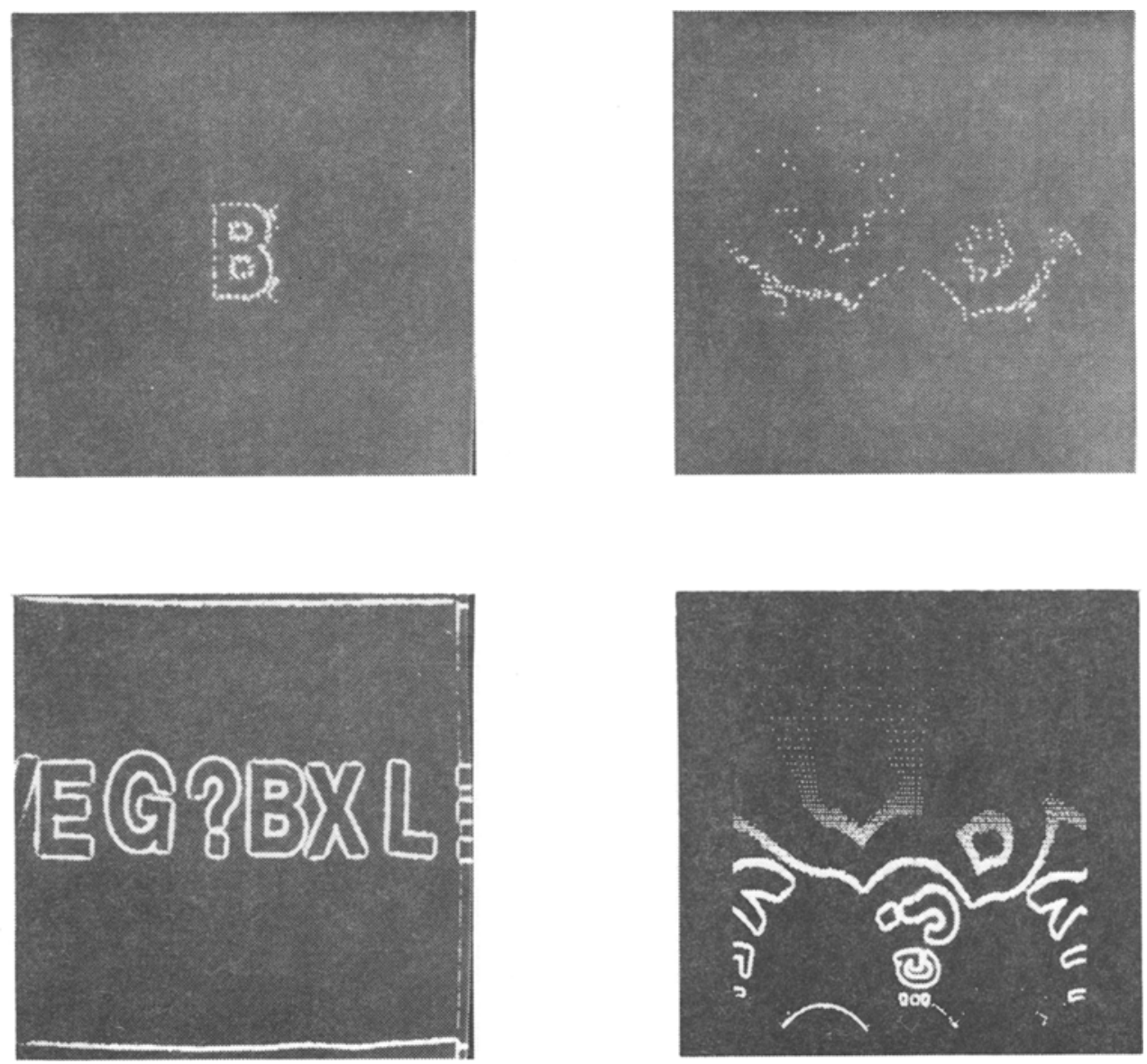

Figure 9. Cartesian edge-only signal (top) and image (bottom) on left, and log-polar versions on right. In the log-polar version, the horizontal axis corresponds to orientation and the vertical axis to log-polar length. The center is chosen with respect to the center of the letter $B$ in both cases.

detectability and decrease false alarms. Furthermore, the use of edge-only information enhances the slope of the correlation image as shown in Figure 8, where the edgeonly Snoopy pattern is detected and localized by the peak of the edge-only cross-correlation function.

\section{Invariance Coding}

Cross-correlation techniques for pattern recognition are shift-invariant insofar as the peak value of Equation 11 is invariant to the location of the signal. It is not invariant, however, to rotation and size changes of the signal. We call the problem of developing techniques to solve this problem, and that of determining how the visual system accomplishes the same, the invariance coding problem, an example of the problem of stimulus equivalence.

Again, there seem to be parallels between solutions to this problem in both areas, and the common idea is that the image is in some way transformed into a log-polar image, as shown for the edge-only image in Figure 9. Such conformal mappings transform rotations and scale changes into translations in the log-polar domain defined by

$$
\begin{gathered}
r^{\prime}=\log \left(r-r_{0}\right) \\
\theta=\tan ^{-1}\left(y-y_{0} / x-x_{0}\right)
\end{gathered}
$$

for $r=\sqrt{x^{2}+y^{2}}, r_{0}=\sqrt{x_{0}^{2}+y_{0}^{2}}$, where $\left(x_{0}, y_{0}\right)$ corresponds to the center of the transform.

As is evident from Equation 13, rotations and size (scale) changes of the pattern become simple translations in the log-polar image, since for:

$$
\begin{gathered}
r_{1}=\alpha\left[r-r_{0}\right), \\
\ln r_{1}=\ln \left(r-r_{0}\right)+\ln \alpha .
\end{gathered}
$$

Also, for rotations, $\theta_{1}=\theta+\phi$. Consequently, a rotation of $\phi$ and scale change of $\alpha$ correspond to a shift of the image by $(\phi, \alpha)$, as indicated in Figure 9 . It should be noted, however, that this solution is not invariant to the choice of center, but does retain the uniqueness of the image. 
Other approaches to this problem involve the use of transform techniques that, in various degrees, retain properties of the image. The closest to the conformal mapping technique discussed above is that of determining the two-dimensional log-polar circular harmonic decomposition of the image (Ferrier, 1986). Essentially, the idea is that if the image is decomposed in terms of concentriccircle/spiral-type "gratings," then the power spectrum of the circular harmonic Fourier spectrum is size- and rotation-invariant. Second, as with the normal Fourier spectrum using vertical gratings, the phase spectrum encodes the actual size and rotation state of the image (Ferrier, 1986): the positional information in this conformal space.

\section{CONCLUSIONS: TECHNICAL IMPLEMENTATIONS}

In Sections 1 through 5, I have presented some recent applications of both image-processing technology and models to the study of human visual information processing. My aim was to demonstrate that a new class of images-naturally occurring achromatic images-can be carefully manipulated and controlled to test many critical hypotheses about information encoding, feature extraction, and pattern recognition. Central to this approach is the image-processing system that allows one to digitize, analyze, and display images in formats up to $512 \times 512$ pixels at relatively little expense.

In the area of image encoding, I briefly examined the question of whether image- or transform-domain-encoding techniques are more efficient or more representative of human image processing. I concluded that the only benefit of spectral representations would be to enable the processes of encoding via the outputs of perceptive fields via enacting convolutions through spectral products (the convolution theorem; see Rosenfeld \& Kak, 1982). I also noted, however, that this encoding required full spectral products and not simply the specification of detectors in the amplitude (power) domain alone. This being the case, then, there would be a formal equivalence between feature detector models and Fourier filters or "channels," since the latter would constitute detectors localized in space and have precisely defined phase responses.

For visual texture segmentation, I examined the idea that texture regions are classified according to the correlation between the responses of detectors within regions. These detectors include the usual edge and bar detectors, and one may determine the objective orthogonality of such textons via the usual definition of zero scalar (dot) product. Such definitions and models endeavor to restrain ambiguous speculations about the detectors involved in texture segmentation.

The issue as to the types of image features that predominate visual information processing, in general, follows naturally from the attempt to define detectors operating in texture segmentation. In Section 3, I examined one important type of image feature: edges.
Research over the past two decades clearly indicates that the "edges" of most interest to human observers are those that are produced from edge and bar detectors extracted from the middle frequency range of an image. Secondly, evidence for edge information from the joint output of detectors enhances the visually predominant edges and also suppresses edge noise contained within each edge filter in isolation. Finally, via relaxation, I showed that pattern-critical features can be extracted without the knowledge of a priori feature lists. Such processes have important implications for pattern recognition based upon the correlation between such features and their spatial distribution.

The difference between these three processes (Sections 1-3 and Table 1) and pattern recognition is that, in the latter, considerable prior knowledge and constraints must apply. This is particularly true with invariance coding and even higher order processes in computational vision. Once pattern features are determined, the signal specified, and the possibilities of log-polar transforms implemented, the recognition of patterns of arbitrary scale, orientation, and position is quite feasible. However, the adaptability of such processes, the learning strategies, and so forth, all require further experimental work before adequate parallels between computer and human vision can be made.

In conclusion, then, I have illustrated how concepts and techniques in computational vision may be applied to the study of human vision. It is my hope that these brief examples may suggest many new experiments and models for human visual information processing. I believe that such image-processing systems allow us to study images that are more "ecologically valid" than the dots, black/white point-plot images, or even gratings that are so frequently used in visual psychophysics.

\section{REFERENCES}

BECK, J. (1966). Perceptual grouping produced by changes in orientation and shape. Science, 154, 538-540.

Braddick, O., Campbell, F., Atkinson, J. (1978). Channels in vision: Basic aspects. In R. Held, H. Leibowitz, \& L. Tenbergen (Eds.), Handbook of sensory physiology: Perception (Vol. 8). Berlin: Springer-Verlag.

Burges, A., \& Ghandeharian, H. (1984). Visual signal detection: I. Ability to use phase information. Journal of the Optical Society of America $A, 1,900-905$.

CAELLI, T. (1983). Energy processing and coding factors in texmure discrimination and image processing. Perception \& Psychophysics, 34, 349-355

CAELLI, T. (1984). On the specification of coding principles for visual image processing. In P. C. Dodwell \& T. Caelli (Eds.), Figural synthesis. Hillsdale, NJ: Erlbaum.

CAELLI, T. (1985). Three processing characteristics of visual texture segmentation. Spatial Vision, 1, 19-30.

Caelli, T., Bevan, P. (1983). Probing the spatial frequency spectrum for orientation sensitivity with stochastic textures. Vision Research, 23, 39-45.

CAelli, T., HüBner, M. (1983). Coding images in the frequency domain: Filter design and energy processing characteristics of the human visual system. IEEE Transactions on Systems, Man \& Cybernetics, SMC-13, 1018-1021. 
CaElli, T., \& HüBNER, M. (1984). On the number of intensity levels detected in textures. Perception, 13, 21-31.

Caelu, T., Julesz, B., \& GiLberT, E. (1978). On perceptual analyzers underlying visual texture discrimination: Part 2. Biological Cybernetics, 29, 201-214.

CaElu, T., \& NaGendran, S. (in press). Fast edge-only matching techniques for robot pattern recognition. Computer Vision, Graphics, \& Image Processing.

CAELLI, T., \& NAWROT, M. (1986). On the localization of signals in images. Manuscript submitted for publication.

CAELlI, T., \& YUZYK, J. (1985). What is perceived when two images are combined? Perception, 14, 14-48.

CAELLI, T., \& YuZYK, J. (1986). On the extraction and alignment of image edges. Spatial Vision, 1, 205-218.

COOLEY, J. W., \& TUKEY, J. W. (1965). An algorithm for the machine calculation of complex Fourier series. Mathematical Computation, 19, 297-301.

Daugman, J. (1984). Spatial visual channels in the Fourier plane. Vision Research, 24, 891-910.

DE VALOIS, R., \& DE VALOIS, K. (1980). Spatial vision. Annual Review of Psychology, 31, 309-341.

FERRIER, N. (1986). Two-dimensional circular harmonic decompositions and recognition of patterns under rotations and size changes. Unpublished master's thesis, University of Alberta, Edmonton, Alberta, Canada.

GeisLER, W., \& HamiLton, D. (1986). Sampling-theory analysis of spatial vision. Joumal of the Optical Society of America A, 3, 62-70.

Hall, E. L. (1979). Computer image processing and recognition. New York: Academic Press.

HubeL, D., \& WeISEL, T. (1977). Functional architecture of macaque monkey visual cortex. Proceedings of the Royal Society, B, 198, 1-59.
Hummel, R., \& Zucker, S. (1983). On the foundation of relaxation labeling processes. IEEE Transactions on Pattern Analysis \& Machine Intelligence, PAMI-5, 267-287,

JULEsz, B. (1962). Visual pattern discrimination. IRE Transactions of the Professional Group on Information Theory, 8, 84-92.

Julesz, B. (1981). Textons, the elements of texture perception, and their interactions. Nature, 290, 91-97.

LiU, Z. Q., \& CAELLI, T. (1986). Adaptive post-filter matching technique for detecting signals in nonwhite noise. Applied Optics, 25, 1622-1626.

MARR, D. (1982). Vision. San Francisco: Freeman.

Minsky, M., PAPERT, S. (1969). Perceptrons: An introduction to computational geometry. Cambridge, MA: MIT Press.

PAPOULIS, A. (1968). Systems and transforms with applications in optics. New York: McGraw-Hill.

Richards, W., Pollit, A. (1974). Texture matching. Kybernetik, 16, 155-162.

Rosenfeld, A., KAK, A. (1982). Digital picture processing. New York: Academic Press.

SeKUler, R. (1974). Spatial vision. Annual Review of Psychology, 25, 292-332.

Treisman, A., \& Gelade, G. (1980). A feature-integration theory of attention. Cognitive Psychology, 12, 97-136.

Watson, A., \& AHUMAdA, A. (1985). Model of human visual-motion sensing. Journal of the Optical Society of America A, 2, 322-342.

Watson, A., Barlow, H., Robson, J. (1983). What does the eye see best? Nature, 302, 419-422.

WATt, R., MORGAN, M. (1985). A theory of the primitive spatial code in human vision. Vision Research, 25, 1661-1674.

Wilson, H., \& GelB, D. (1984). Modified line-element theory for spatial-frequency and width discrimination. Joumal of the Optical Society of America A, 1, 124-131. 